\title{
SUSTENTABILIDADE EM CLUSTERS: PROPOSIÇÃO DE UM MODELO CONCEITUAL
}

SUSTAINABILITY IN CLUSTERS: PROPOSAL OF A CONCEPTUAL MODEL

SOSTENIBILIDAD EN CLUSTERS: PROPUESTA DE UN MODELO CONCEPTUAL

\section{Fabiano Larentis}

Doutor em Administração pela Universidade Federal do Rio Grande do Sul - UFRGS

Professor da Universidade de Caxias do Sul - UCS

E-mail: flarenti@ucs.br (Brasil)

\section{Rafael Giovanella}

Mestrando em Administração pela Universidade de Caxias do Sul - UCS

Professor da Faculdade América Latina - FAL

E-mail: giovanellar@gmail.com (Brasil)

\section{Tatiane Pellin Cislaghi}

Mestre em Administração pela Universidade de Caxias do Sul - UCS

Professora do Instituto Federal de Educação, Ciência e Tecnologia do Rio Grande do Sul - IFRS

E-mail: tatiane.cislaghi@bento.ifrs.edu.br (Brasil) 


\section{SUSTENTABILIDADE EM CLUSTERS: PROPOSIÇÃO DE UM MODELO CONCEITUAL}

\section{RESUMO}

A crescente reestruturação produtiva e os movimentos de cooperação aumentam as pressões por redução de custos e incremento de produtividade, com isso acaba-se por gerar a formação de novos arranjos entre as empresas, com ênfase nos clusters. Os clusters formam-se quando os aspectos setorial e geográfico estão concentrados e quando a especialização e a inovação são essenciais para que as empresas atinjam mercados além de suas fronteiras. Por outro lado, um dos desafios no desenvolvimento de um cluster está relacionado à sua sustentabilidade, nos aspectos econômico, social e ambiental. Desta maneira, neste ensaio teórico, propõe-se um modelo conceitual que aborda a relação entre clusters e sustentabilidade, levando em consideração a perspectiva de redes. Tal modelo, em formato de espiral, envolve aspectos de base (atores, mecanismos de governança, recursos e localização), intermediários (força e abertura do cluster, processos de integração e adaptação, criação/estoque e movimentação de conhecimento, ações de utilização e de exploração) e de resultados (desempenho e sustentabilidade do cluster), em um contexto de ciclo de vida e da dinâmica ambiental.

Palavras-chave: Clusters; Desenvolvimento Sustentável; Redes.

\section{SUSTAINABILITY IN CLUSTERS: PROPOSAL OF A CONCEPTUAL MODEL}

\section{ABSTRACT}

The growing industrial restructure and movements of cooperation, increase pressures by reducing costs and increasing productivity, thus it has generated the formation of new arrangements between the companies, with focus on clusters. The clusters are formed when the similar areas and geographical aspects are concentrated and where the expertise and innovation are essential for companies to reach markets beyond their borders. On the other hand, one of the challenges in developing a cluster is related to its sustainability, in the economic, social and environmental dimensions. Thus, in this essay, we propose a conceptual model that addresses the relationship between clusters and sustainability, taking into account the perspective of networks. Such a model, spiral shaped, involves basic (actors, governance mechanisms, resources and location), intermediate (cluster strength and openness, integration and adaptation, knowledge creation / inventory and movement, exploitation and exploration actions) and resulting aspects (performance and sustainability of the cluster), in a context of life cycle and environmental dynamics.

Keywords: Clusters; Sustainable Development; Network. 
Fabiano Larentis, Rafael Giovanella \& Tatiane Pellin Cislaghi

\section{SOSTENIBILIDAD EN CLUSTERS: PROPUESTA DE UN MODELO CONCEPTUAL}

\section{RESUMEN}

La reestructuración productiva y el aumento de los movimientos cooperativos aumentan la presión para la reducción de costos y aumento de la productividad, por lo que terminan generando la formación de nuevos acuerdos entre las empresas, con énfasis en los grupos. Los grupos se forman cuando se concentran los aspectos sectoriales y geográficos y donde la experiencia y la innovación son esenciales para que las empresas lleguen a los mercados más allá de sus fronteras. Por otro lado, uno de los retos en el desarrollo de un cluster está relacionada con su sostenibilidad en los aspectos económicos, sociales y ambientales. Por lo tanto, este trabajo teórico, se propone un modelo conceptual que aborda la relación entre los clusters y la sostenibilidad, teniendo en cuenta la perspectiva de las redes . Este modelo, en forma de espiral, involucra los aspectos básicos (actores, mecanismos de gobernanza, recursos y ubicación), intermedio (la fuerza y la apertura de la agrupación, los procesos de integración y adaptación, creación/almacenamiento y manejo de conocimientos, acciones y uso operación) y resultados (rendimiento y la sostenibilidad del clúster), en el contexto del ciclo de vida y la dinámica ambiental .

Palabras-clave: Clusters; El Desarrollo Sostenible; Networks. 


\section{INTRODUÇÃO}

Mudanças na tecnologia, estruturas políticas e sociais, bem como os impactos da globalização têm colocado pressão sobre os países ou regiões para se tornarem competitivos. Um mecanismo de crescimento econômico encontrado para suprir tal necessidade é a criação de clusters (Calamel, Defélix, Picq, \& Retour, 2012).

Como uma configuração interorganizacional complexa e multifacetada, cluster é uma concentração regional de empresas relacionadas e interconectadas (Aziz \& Norhashim, 2008; Pedrozo \& Hansen, 2001; Perry, 2005; Porter, 1998). Clusters bem sucedidos não apenas atingem resultados positivos por uma estrutura de apoio qualificada, de inovação, aprendizagem compartilhada, visão de mercado e ações governamentais adequadas, mas atingem também porque conseguiram bons relacionamentos e cooperação entre as empresas e outras organizações integrantes (Bell, 2005; Giuliani \& Bell, 2005; Porter, 1998). Ou seja, por terem desenvolvido elementos presentes em uma perspectiva de rede.

Por outro lado, o cluster possui um papel e uma atuação na região em que está inserido, não somente econômica, mas também de caráter social e ambiental. Em relação a isso, como indicam Cunha e Cunha (2005), um dos desafios para os agentes responsáveis pela elaboração e implementação de políticas de desenvolvimento de um cluster é construir um modelo de desenvolvimento sustentado com competitividade, sustentabilidade e justiça social.

Desta forma, tendo como base a perspectiva de redes, o presente ensaio teórico tem como objetivo propor um modelo conceitual derivado da relação entre cluster e sustentabilidade. Esse artigo está estruturado da seguinte forma: primeiramente apresenta-se a questão do desenvolvimento sustentável. A seguir, a relação de clusters com redes e com sustentabilidade e o modelo conceitual proposto. Por fim, algumas considerações finais acerca do tema.

\section{DESENVOLVIMENTO SUSTENTÁVEL E ESTRATÉGIAS SUSTENTÁVEIS}

Um olhar profundo nos ensinamentos das diferentes religiões, filosofias medievais e crenças tradicionais revela que, apesar das variações semânticas, a maior parte delas contém um componente forte de viver em harmonia com a natureza e entre seres vivos. Essa é a essência lógica do que se chama hoje de sustentabilidade (Mebratu, 1998). Considerando isso, desenvolvimento sustentável é aquele, portanto, que alcança as necessidades das gerações atuais sem comprometer a habilidade de gerações futuras de alcançar suas necessidades e aspirações (Steurer, Langer, Konrad, \& Martinuzzi, 2005). 
Fabiano Larentis, Rafael Giovanella \& Tatiane Pellin Cislaghi

Tradicionalmente, conforme Steurer et al. (2005), o desenvolvimento sustentável, que é um conceito orientado para processos, está baseado em três dimensões: a econômica (desempenho financeiro, competitividade de longo prazo, impacto econômico), a social (equidade: distribuição de renda mais justa dentro da organização e entre unidades de locais diferentes, assim como melhorias sociais internas e externas) e a ambiental (uso de recursos, emissões, danos e riscos ambientais).

Todavia, ainda conforme Steurer et al., há uma quarta dimensão com questões de segunda ordem, como (a) transparência e participação (abertura da empresa), (b) reflexividade (aprendizado contínuo através de monitoramento e avaliações), (c) integração (progresso em uma dimensão não pode ocorrer às custas de outra dimensão) e (d) equidade intergerações (satisfazer as necessidades da empresa e de seus stakeholders hoje e no futuro).

Sustentabilidade nos negócios é encarada, portanto, não como uma iniciativa ambiental, mas como uma estratégia empresarial que gera valor a partir da busca de melhores resultados sociais e ambientais (Benites \& Pólo, 2013). Nesse ínterim, para Cruz, Pedrozo e Estivalete (2006), possivelmente uma das demandas mais complexas e importantes que vêm ocupando as reflexões dos gerentes é a incorporação do desenvolvimento sustentável nessas estratégias. Há preocupações, tanto em relação aos acionistas quanto aos stakeholders. Surgem trade-offs a ser analisados e julgados. Nesse aspecto, considerando o papel da organização, eles questionam se ela hoje existe para satisfazer objetivos individuais e societais como um todo ou os indivíduos e a sociedade existem como um todo para permitir o alcance de objetivos organizacionais.

Os autores sugerem que "lentes" tradicionais, baseadas na lógica econômico-financeira, não dão conta suficientemente da compreensão e da prática do desenvolvimento sustentável. Para isso sugerem a perspectiva da complexidade de Morin, que permite de maneira mais abrangente observar a passagem dessa lógica tradicional para uma sustentável. Devido ao caráter multifacetado, complexo da realidade, o paradigma da complexidade introduz uma análise interdisciplinar do fenômeno. Procura eliminar o ponto de vista reducionista que analisa o fenômeno sem considerar as repercussões sistêmicas envolvidas.

É nesse contexto que surgem, como necessidade, as estratégias sustentáveis, ou seja, como as possibilidades das ações estratégicas podem levar à noção de desenvolvimento sustentável, à incorporação de uma análise multidimensional e a consideração de múltiplos stakeholders. Estratégia sustentável, dentro de uma perspectiva complexa e de longo prazo ou, em outras palavras, dependente de uma visão compartilhada do futuro (Hart, 1995).

Para as empresas pensarem suas estratégias como uma prática social, é necessário que não apenas haja consideração a impactos econômicos, sociais e ambientais atrás de suas ações, mas também um avanço em suas relações, mudando de uma lógica de acionista a uma lógica de 
stakeholder: bons resultados financeiros são buscados de tal forma que os stakeholders não necessariamente saiam perdendo. É preciso considerar o impacto das ações estratégicas na empresa que as executa e na sociedade como um todo (Cruz et al., 2006).

Por outro lado, quanto mais stakeholders sérios e comprometidos apoiarem conceitos como desenvolvimento sustentável e responsabilidade social corporativa, mais esses conceitos conseguirão acesso às atividades corporativas centrais, notadamente através da gestão das relações com os mesmos (Steurer, et al., 2005). Todavia, de acordo com Cruz et al. (2006), passar de uma lógica financeira para uma lógica sustentável não ocorre imediatamente. É necessário, segundo eles que a organização passe por um processo de aprendizagem como um todo, assim avançando para uma orientação sustentável.

Nesse aspecto, as ações calcadas na perspectiva sustentável não surgiriam somente por exigências institucionais (incluindo regulamentos e imagem) ou de mercado (incluindo decisões de investidores dependentes de práticas sustentáveis) (Bansal \& Roth, 2000; Cruz, et al., 2006; Mackey, Mackey, \& Barney, 2007), mas seriam acompanhadas por mudanças nos valores dos envolvidos, as quais poderiam impactar as suas relações inclusive fora das empresas onde trabalham, influenciando suas comunidades (Bansal \& Roth, 2000; Cruz et al., 2006). Avançar na lógica orientada à sustentabilidade significa engajar os indivíduos nesse propósito e torná-los disseminadores potenciais desses valores, assim criando um movimento de mudança na sociedade como um todo, já que para uma visão de mundo ser congruente como desenvolvimento sustentável, deve manifestar inclusividade, conectividade, equidade, prudência e seguridade (Gladwin, Kennelly, \& Krause, 1995; Cruz et al., 2006).

Da perspectiva organizacional, Schaltegger e Wagner (2011) ressaltam o aumento da importância das questões atinentes ao desenvolvimento sustentável na gestão das organizações. Segundo eles, há inovações que demandam objetivos ambientais e sociais, que podem ser bem sucedidos em mercados tradicionais. Na empresa orientada para ações de sustentabilidade, a principal característica é o esforço para reduzir os impactos sociais e ambientais, via adaptação de produtos, processos e estruturas organizacionais.

Em relação a isso, Mauerhofer (2008) apresenta o modelo do Triângulo 3-D da Sustentabilidade, o qual considera as hierarquias e prioridades relacionadas à sustentabilidade ambiental, social e econômica. Nesse modelo, o triângulo reflete a biosfera global e sua capacidade total de resiliência e limites. A base do triângulo é formada por três círculos sobrepostos: capital natural, o externo; capital social, o intermediário; e capital econômico, o interno. Em cada um desses círculos, há um pilar, retratando a capacidade e uso dos ativos de cada capital: capacidade 
Fabiano Larentis, Rafael Giovanella \& Tatiane Pellin Cislaghi

ambiental, social e econômica. Estes pilares sustentam um triângulo menor, o qual representa o equilíbrio ou desequilíbrio da sustentabilidade como um todo.

Com base nisso, Marconatto, Trevisan, Pedroso, Saggin e Zonin (2013) apresentam um modelo conceitual analítico-decisório de três fases: (1) análise das demandas dos diversos stakeholders frente à organização; (2) esforço de incorporação dessas demandas no sistema de negócios da organização, a partir das prioridades da sustentabilidade (hierarquia) e dos princípios da ecologia industrial; (3) análise dos impactos do novo sistema de negócios no Triângulo 3-D de Sustentabilidade. De acordo com os autores, provavelmente mudanças organizacionais alterem o equilíbrio interno do Triângulo 3-D, já que poderá haver alterações na relação entre os capitais e as capacidades econômicas, sociais e ambientais. A reconfiguração desses elementos, por outro lado, poderá implicar em mudanças na organização e nos interesses dos stakeholders. Tais modelos retratam, portanto, a relevância, mas também a complexidade de se colocar em prática estratégias sustentáveis.

\section{CLUSTER}

O conceito mais popular é o conceito proposto por Porter (1998). Ele definiu cluster como concentrações geográficas de empresas interconectadas, fornecedores especializados, prestadores de serviços, empresas em setores relacionados e instituições associadas em áreas específicas que competem, mas também cooperaram e como uma forma de rede que ocorre dentro de uma região geográfica, em que a proximidade de empresas e instituições garante certas formas de comunhão e aumenta a frequência e o impacto das interações.

Complementos à definição de Porter são sugeridos por Rosenfeld (1997), que destacou a importância da capacidade de produzir sinergia, entre as organizações. Feser (1998) sugere que o que define um cluster não é apenas as empresas, mas também as instituições de apoio, como instituições de ensino, associações empresariais e centros de pesquisa. Roelandt e Hertog (1999) descrevem cluster como uma cadeia de produção de valor acrescentado.

Em um cluster, há campos que competem, mas que também cooperam: há existência de similaridades e complementaridades, o sistema de empresas e instituições interconectadas faz com que o valor como um todo seja maior que a soma das partes (Baptista \& Swan, 1998; Porter, 1998). Nesse sentido, Zaccarelli, Telles, Siqueira, Boaventura e Donaire (2008) afirmam que o conjunto de empresas de um cluster forma um sistema, que pode ser compreendido como uma entidade supra- 
empresarial, e que, embora possa ser considerada uma abstração, apresenta comportamento com características próprias, que não existem isoladamente nas empresas.

Conforme Perry (2005), cluster apresenta principalmente uma condição de localização particular e, ao mesmo tempo, pode ser classificada como economia de alto desempenho. Quanto a isso, indica que os negócios competem entre si por participação de mercado, empregados e recursos, até mesmo mais vigorosamente do que aqueles que estão fora do cluster. Por outro lado, os negócios dependem um dos outros.

Em resumo, de acordo com Aziz e Norhashim (2008), cluster é definido como um conjunto de atores (empresas de ao menos um setor, agências e instituições) concentrados geograficamente, com similaridades, complementaridades e relacionamentos tanto competitivos quanto cooperativos. Além de diversas atividades econômicas, passa por aquelas com significativo nível de conhecimento e de tecnologia, promovendo entre seus membros transferências e propagação (spillover). Coletivamente, tem um impacto significativo sobre os níveis econômicos maiores (regional ou nacional). Fica evidente o foco na localização e o aspecto de regionalização (Pedrozo \& Hansen, 2001).

A aglomeração de empresas é um fenômeno antigo, havendo, inclusive, registros datando da Idade Média. No Brasil, popularizou-se o termo Arranjo Produtivo Local (APL), como indicam Mascena, Figueiredo e Boaventura (2013). Para Brito e Stallivieri (2010), APL constitui-se em uma aglomeração espacial de agentes econômicos, políticos e sociais envolvidos com um conjunto específico de atividades produtivas, na qual se estruturam vínculos e relações de interdependência. Por outro lado, segundo Galdámez, Carpinetti e Gerolamo (2009), APLs promovem a cooperação, a inovação contínua e o desenvolvimento sustentável de pequenas e médias empresas, sendo necessário construir uma infra-estrutura local e um ambiente que estimule a confiança e a cooperação dos membros.

Em relação a isso, Aquino e Bresciani (2005) comentam que o termo APL pode ser tomado como tradução do termo cluster: o essencial da definição está na especialidade da produção e na delimitação espacial. O termo APL pode ser entendido como um sinônimo para o termo cluster, como encontra-se na literatura internacional. No entanto, na visão de Figueiredo e Di Sério (2007), os clusters se diferenciam dos APLs pela maior intensidade de vínculos entre as empresas e pelo nível de participação dentro do agrupamento, com menor envolvimento do governo, enquanto que no APL o poder público e agências de fomento são mais atuantes.

Para Aquino e Bresciani (2005) e Masquietto, Neto e Giuliani (2010), os APLs constituem, acima de tudo, uma diretriz importante no âmbito das políticas públicas. Isto porque determinadas 
Fabiano Larentis, Rafael Giovanella \& Tatiane Pellin Cislaghi

ações e estratégias associadas aos APLs constituem alternativas para o aumento da competitividade ao desenvolvimento econômico e social, regional e nacional.

Nesse sentido, conforme pesquisa bibliométrica das publicações nacionais de 2000 a 2011 de clusters e APLs, desenvolvida por Mascena, Figueiredo e Boaventura (2013), muitos dos artigos analisados referem-se às políticas públicas e ao papel das instituições como propulsoras do surgimento e desenvolvimento dos clusters. Segundo eles, a partir do contexto brasileiro, onde muitos clusters surgiram especialmente em forma de APLs como uma estratégia para promover o desenvolvimento local, muitos estudos destacam a importância de agentes externos. A pesquisa também ressalta o aumento de trabalhos associados à questão da cooperação, utilizando como embasamento teórico temas de redes sociais, aprendizado, capital social e ação coletiva.

A seguir, então, as características e desenvolvimento dos clusters, relação dos clusters com redes e desempenho de clusters.

\subsection{CARACTERÍSTICAS E DESENVOLVIMENTO DOS CLUSTERS}

Mesmo com a globalização e mudança no poder dos atores envolvidos, os clusters não perdem espaço; na realidade, ganham (Porter, 1998). Isso pela concorrência moderna depender em alto grau da produtividade e não apenas de acesso a insumos ou da economia de escala de empreendimentos isolados. Esta produtividade é dependente do grau de sofisticação da gestão das empresas, o qual é fortemente influenciada pelas condições do ambiente empresarial local vinculado aos diferentes clusters (Pedrozo \& Hansen, 2001).

Segundo Porter (1998), o cluster consiste de uma combinação de produtos finais, maquinário, materiais e serviços associados. Suas fronteiras desenvolvem-se com novas empresas que se estabelecem, com novas tecnologias e marcos regulatórios surgindo. Para explicar as estrutura e dimensões do cluster, o autor sugere a metáfora do diamante, considerando como seus vértices (a) o contexto para estratégia e rivalidade (que encoraja investimento e competição vigorosa); (b) as condições de demanda (clientes e necessidades - incluindo clientes locais); (c) as organizações relacionadas e de apoio (fornecedores de insumos e serviços) e (d) as condições dos fatores (recursos naturais, humanos, capitais, infra-estrutura, qualidade e especialização). Os clusters são vistos melhor como manifestações das interações entre essas quatro facetas (vértices).

Semelhante ao diamante proposto por Porter, Huggins (2008), baseado na tipologia de Tödtling (1994) e com enfoque nas empresas intensivas de conhecimento, apresenta cinco dimensões associadas aos clusters: (a) empresas do cluster; (b) mercados (clientes regionais, nacionais e internacionais); (c) empresas de apoio (fornecedores de bens e serviços); (d) entradas de 
fatores (capital de risco, habilidades de gestão e técnicas, conhecimento e tecnologia); (e) políticas públicas (infraestrutura, regulação, instituições de ensino, transporte e comunicação, políticas de tecnologia). Tais dimensões são influenciadas pelos ambientes local e global, pela presença de graduados, consultores, empreendedores, empresas e investidores, movimentação da mão-de-obra, fornecimento de bens de capital, colaboração em pesquisa, desenvolvimento e inovação, gestão legal e novas tecnologias e inovações.

Porter reforça, nesse ínterim, que o teste final para a saúde de um cluster é sua taxa de inovação. Empresas em clusters percebem mais claramente e rapidamente novas necessidades dos clientes e novas necessidades de tecnologias, há flexibilidade e capacidade em agir rapidamente. Por outro lado, pode haver retardo da inovação, dependendo da inflexibilidade resultante de comportamentos uniformes dentro do cluster, da distância tecnológica e das complementaridades entre as empresas (Baptista \& Swan, 1998; Porter, 1998).

Outro ponto importante para os clusters é o papel dos governos. Porter (1998) aponta que estes devem reforçar o desenvolvimento de todos os clusters, não escolher um em específico; permitir condições econômicas e sociais para o surgimento e desenvolvimento deles e trabalhar nos já estabelecidos e que estão surgindo, não tentar criar alguns, intervindo por completo. Além disso, a existência de clusters cria novos papéis ao governo.

Já Rosenfeld (1997) indica que os governos devem aprender como negócios interagem e clusters funcionam, melhorar os serviços de apoio técnico, investir em capital social (o estoque de conexões ativas entre as pessoas, possibilitando a cooperação entre as pessoas) e infra-estrutura social (incentivos para ativar associações atuais ou novas), dar poder e escutar os líderes do cluster, encorajar a fertilização cruzada de ideias, recrutar empresas que podem preencher vazios no desenvolvimento do cluster, desenvolver e organizar associações de cadeia de suprimentos e apoiar empreendedores e empregados. Comparado a Porter, Rosenfeld apresenta um governo com papel mais ativo.

Considerando, paralelamente, as fases do surgimento, desenvolvimento e declínio dos clusters, Porter indica como causas para surgirem a disponibilidade de fatores (habilidades especializadas, expertise em pesquisa, locações físicas eficientes, infra-estrutura adequada); demanda sofisticada; existência de fornecedores relacionados ou outros clusters; empresas inovadoras que estimulam o crescimento de outras; e eventos aleatórios. Alerta, todavia, que a mera presença de organizações em um local cria o potencial de valor econômico, mas não necessariamente garante a sua realização. Nesse sentido, Rosenfeld (1997) apresenta como clusters potenciais aqueles que têm sistemas de produção e social incompletos, mas com os ingredientes 
Fabiano Larentis, Rafael Giovanella \& Tatiane Pellin Cislaghi

básicos para se desenvolver como sistema. Por outro lado, conforme Giuliani e Bell (2005), empresas podem se manter geograficamente próximas mas não cognitivamente associadas: o cluster não dever ser somente localizado, mas também ser sentido como tal.

Meyer-Stamer (2001), por sua vez, apresenta como fatores necessários ao surgimento de clusters os de ordem objetiva e os de ordem subjetiva. Os fatores objetivos são a posição geográfica em relação aos mercados de compra e venda, infraestrutura disponível (transporte, energia, comunicações), mão-de-obra, disponibilidade de terrenos, impostos, incentivos oferecidos pelos governos. Já os fatores subjetivos são o ambiente econômico da região correspondente, imagem da região, os contatos setoriais, com universidades e instituições de pesquisa, o perfil inovador da região, o desempenho de associações de classe e qualidade de vida da região (educação, renda, infraestrutura, meio ambiente, lazer e cultura).

$\mathrm{Na}$ fase do desenvolvimento, Porter indica que a eficácia dos clusters depende de como estão constituídos e ser relacionam os vértices do diamante. Por outro lado, argumenta que o desenvolvimento dos clusters frequentemente torna-se particularmente vibrante em suas intersecções com outros. Além disso, coloca que o desenvolvimento dos clusters leva à sua internacionalização e reforça a necessidade da atração de pessoas e ideias que o intensificam.

Quanto ao declínio, o autor apresenta como razões as variáveis endógenas e exógenas. As endógenas são as de ordem interna, como a inflexibilidade. As exógenas representam descontinuidades do ambiente, como a tecnologia. Porter indica, em relação a isso, que o teste final para a saúde de um cluster é sua taxa de inovação. Por outro lado, segundo Meyer-Stamer (2001), é frequente o fracasso de ações de estímulo à cooperação entre empresas do mesmo setor numa determinada cidade ou região, dado o problema da falta de confiança.

De maneira análoga, Aziz e Norhashim (2008) apresentam um modelo de clusters em seis estágios, considerando seu ciclo de vida, cujo aspecto chave para avaliá-lo é a tecnologia dominante e sua trajetória: (a) antecedência, (b) cluster embrionário, (c) cluster em desenvolvimento, (d) cluster maduro, (e) cluster em declínio ou (f) transformação (Quadro 1): 


\begin{tabular}{|c|c|}
\hline ESTÁGIOS & CARACTERÍSTICAS \\
\hline Antecedência & $\begin{array}{l}\text { Momento inicial que mostra ou ajuda a dar o impulso para formar o cluster. Pode ser através de } \\
\text { um desenvolvimento orgânico (tendências naturais) ou planejado (decisões foram feitas para } \\
\text { obter os benefícios e foram conduzidos através de implementações de políticas). }\end{array}$ \\
\hline $\begin{array}{l}\text { Cluster } \\
\text { embrionário }\end{array}$ & $\begin{array}{l}\text { Quando o cluster apresenta sinais de economias de aglomeração e os atores estão se } \\
\text { beneficiando dele, bem como começa a formar ativamente ligações e redes. }\end{array}$ \\
\hline $\begin{array}{c}\text { Cluster em } \\
\text { desenvolvimento }\end{array}$ & $\begin{array}{l}\text { Massa crítica foi atingida e as ligações são ativas dentro do cluster. As relações com partes } \\
\text { externas estão sendo desenvolvidas. }\end{array}$ \\
\hline Cluster maduro & $\begin{array}{l}\text { O grupo atingiu seu nível máximo e seu setor chave ou tecnologia amadureceu. Crescimento e } \\
\text { desempenho mostram uma acentuada desaceleração. }\end{array}$ \\
\hline $\begin{array}{l}\text { Clusters em } \\
\text { declínio: }\end{array}$ & $\begin{array}{l}\text { Passam pela maturidade e têm dificuldade em encontrar novo foco para gerar um novo } \\
\text { crescimento e para atrair novos participantes, úteis para reenergizar a sua composição. Significa } \\
\text { que o cluster pode se desintegrar, com atores procurando novos clusters emergentes ou locais } \\
\text { com melhores economias de escala. }\end{array}$ \\
\hline Transformação & $\begin{array}{l}\text { É quando o cluster maduro ou em declínio está mostrando sinais de um novo crescimento, } \\
\text { envolvendo por exemplo novos participantes para o cluster, novas tecnologias e novos } \\
\text { segmentos de mercado, através de políticas públicas e de inovação ou a inserção de novo(s) } \\
\text { grande(s) entrante(s). Alguns atores podem sair, mas o limite de massa crítica é mantida (com } \\
\text { adição de novas empresas). O cluster parte para um novo ciclo de vida, dependendo de qual } \\
\text { estágio irá se reinserir de acordo com sua trajetória. }\end{array}$ \\
\hline
\end{tabular}

Quadro 1 - Estágios do ciclo de vida de um cluster

\subsection{CLUSTERS E REDES}

Redes de organizações podem ser indicadas como qualquer conjunto de atores (maior ou igual a dois) que buscam relações de trocas repetidas e duradouras e, ao mesmo tempo, onde não há evidente uma autoridade organizacional legítima para arbitrar e resolver disputas que podem surgir (diferentemente das hierarquias) (Polodny \& Page, 1998). Além disso, a capacidade de manter e construir relacionamentos de redes interorganizacionais é cada vez mais vista como chave para vantagem competitiva sustentável, em especial devido à possibilidade de gerar e sustentar o desenvolvimento e a competitividade de empresas e regiões (Omta, Trienekens, \& Beers, 2001; Verschoore \& Balestrin, 2008).

Neste sentido, destacam Verschoore e Balestrin (2008) que o propósito central das redes é reunir atributos que permitam uma adequação ao ambiente competitivo em uma única estrutura, 
Fabiano Larentis, Rafael Giovanella \& Tatiane Pellin Cislaghi

sustentada por ações uniformizadas, porém descentralizadas, que possibilite ganhos de escala sem perder a flexibilidade por parte das empresas associadas. Trata-se, dessa forma, de um modo de associação por afinidade de natureza predominantemente informal e que deixa cada uma das empresas responsável pelo seu próprio desenvolvimento (Olave \& Neto, 2001).

Tendo em vista as diversas formas de governança, de acordo com Chung, Yam e Chan (2004), enquanto que no mercado os principais mecanismos de coordenação (governança) são a barganha e competição e na firma (hierarquia) a autoridade e a identificação, na rede são a negociação e a concorrência. Por outro lado, considerando os fluxos de informação, no mercado estão confinados aos termos da troca enquanto que na firma e na rede há um alto grau de compartilhamento de informação com relação a um espectro maior de informação.

Como benefícios, redes permitem, aos envolvidos, aprendizagem, legitimação e status, benefícios econômicos, autonomia, menor desigualdade na distribuição de riqueza, ampliação do capital social da empresa (fica mais fácil conseguir informações, conhecimento técnico e apoio financeiro) e maior senso de comunidade e da responsabilidade/dever social (reduzindo possibilidades de se relacionar com companhias fora da rede) (Polodny \& Page, 1998; Omta, Trienekens, \& Beers, 2001). Todavia, exigem formas de governança capazes de conciliar competição e cooperação, relações de poder, autonomia e interdependência para o desenvolvimento sinérgico das competências dos seus membros (Quandt, 2012).

Assim, partindo da relação de redes com clusters, Meyer-Stamer (2001) indica que, especialmente nos distritos industriais italianos (aglomeração industrial com perfil restrito de especialização), é comum a cooperação entre empresas, no que se refere à cadeia de valor e aos segredos empresariais. Segundo ele, condições de entrega e outras formas de cooperação entre empresas podem ser regulamentadas informalmente enquanto o capital social cria a condição para as relações confidenciais, e abre a possibilidade de tomar medidas de emergência contra um procedimento oportunista. Isso está presente de forma semelhante, conforme Pedrozo e Hansen (2001), nas redes flexíveis de pequenas e médias empresas, que considera, em certo grau, a regionalização, pelo porte das empresas analisadas.

Porter (1998), por sua vez, indica a importância da existência de uma estrutura social com relacionamentos, comunicação aberta e senso de interesse comum, ou seja, o cluster sob um prisma de rede, que ocorre em um local geográfico, onde a proximidade de empresas e instituições garante certas formas de comunalidades e aumenta a frequência e impacto das interações. Segundo, Aziz e Norhashim (2008), relações tênues podem se fortalecer simplesmente através do poder de proximidade. 
Além disso, levando em conta redes internas ao cluster, de acordo com Bell (2005), empresas e executivos estão envolvidos em dois tipos: uma rede gerencial de ligações informais e uma rede institucional de ligações formais entre empresas. A rede gerencial amplia o fluxo de informações, incluindo as tácitas, e depende de um contexto de alta confiança para se comunicar. A rede institucional, por sua vez, fornece oportunidades para informar-se a respeito de notícias da indústria. Nesse sentido, ele identificou que a centralidade gerencial (exposição a mais informações, forte envolvimento na rede) influencia mais a inovação que a centralidade institucional.

Como indicam Aziz e Norhashim (2008), em um cluster pode haver o benefício de relacionamentos tanto cooperativos quanto competitivos, de haver concorrentes que participam de atividades colaborativas (a lógica da coopetição). Neste ínterim, em um estudo avaliando práticas de marketing em cluster, Felzensztein, Gimmon e Aqueveque (2012) identificaram que gerentes de empresas que fazem parte de cluster tendem a perceber mais benefícios e oportunidades para a cooperação interorganizacional em atividades de marketing. Os autores sugerem que os gestores não devem desconfiar da cooperação, tanto a nível vertical quanto horizontal, pela contribuição no desenvolvimento de vantagens competitivas.

Portanto, mesmo que o cluster enfatize principalmente o elemento localização e a concentração de empresas, onde podem estar presentes tanto questões de competição como de cooperação, e que nas redes considera-se principalmente ações de cooperação baseadas na confiança e nos relacionamentos duradouros entre as empresas, pode-se perceber os clusters numa perspectiva de redes. Como exposto anteriormente, diversos clusters se beneficiaram por considerar em sua constituição e desenvolvimento elementos característicos de redes.

\subsection{DESEMPENHO DOS CLUSTERS}

O desempenho de um cluster pode ser observado na inovação produzida por seus atores, envolvendo o nível de sucesso na comercialização dos bens produzidos, bem como a criação de empresas no cluster, no nível de empregos e nos resultados financeiros de saída, controlando a taxa de crescimento nacional dessas medidas no setor relevante (Aziz \& Norhashim, 2008; Eisingerich, Bell, \& Tracey, 2010).

De forma mais abrangente, conforme Carpinetti, Galdámez e Gerolamo (2008), há quatro perspectivas de desempenho do cluster: (a) resultados econômicos e sociais (relacionados com o produto interno bruto local, força de trabalho, pessoal treinado, ocupação e outros itens que indiquem benefícios econômicos e sociais); (b) desempenho das empresas (crescimento e 
Fabiano Larentis, Rafael Giovanella \& Tatiane Pellin Cislaghi

competitividade das empresas, através de indicadores financeiros e não-financeiros, como produtividade, aumento nas vendas e do valor de mercado); (c) eficiência coletiva (economias externas/reduções coletivas de custo, níveis de produtividade e ações de cooperação entre as empresas do cluster); (d) capital social (valores culturais, tais como confiança e cooperação) e (f) impacto ambiental (medidas de sustentabilidade ambiental).

Em relação a isso, tais resultados dependerão da capacidade de seus membros de reconfigurar as relações para atender à emergente demanda do mercado e de incorporar as mudanças em tecnologia (Eisingerich et al., 2010). Para tanto, Aziz e Norhashim (2008) propõem, como forma de compreender o desempenho de um cluster como variável dependente, a Pirâmide de Desempenho do Cluster (PDC). No seu topo (primeiro nível) está o desempenho do cluster. O segundo nível é composto pela (a) criação e estoque (repositório) de conhecimento e (b) pela movimentação do conhecimento, itens considerados como primeiro conjunto de variáveis independentes. Abaixo disso, como aspectos de base, se apresentam os atores do cluster (terceiro nível) e a dinâmica do cluster (quarto nível).

Segundo eles, a criação e estoque de conhecimento é um dos aspectos que determina o nível da sua inovação e da eventual comercialização dos bens e novas empresas, que pode ser avaliado a partir dos valores financeiros das atividades associadas realizados, principalmente em pesquisa e desenvolvimento. $\mathrm{O}$ estoque de conhecimento é incorporado pelos produtos, processos, tecnologias e trabalhadores do conhecimento das organizações do cluster.

Por outro lado, a movimentação do conhecimento é o segundo conjunto de variáveis independentes que influencia o desempenho do cluster, composto pelas interações, integração e cooperação dos atores dentro do cluster. Tal aspecto pode ser avaliado através do número de meios de trabalho colaborativo promovido pelos atores do cluster, a existência e nível de utilização de plataformas de compartilhamento de conhecimentos, tais como comunidades on-line, conferências e simpósios e, por fim, o movimento de empregados (portadores de conhecimento / criadores) entre os atores dentro do cluster. Estes fazem-se o segundo conjunto de independentes variáveis.

Todavia, a criação e estoque de conhecimento e os aspectos do movimento de conhecimento são apenas de interesse quando ambos são realizados por organizações dentro do cluster, ou seja, os atores. É reconhecido a partir da literatura que os principais tipos de atores que têm mais impactos significativos ao sistema do cluster e podem ser encontrados em quaisquer agrupamentos são indústrias, comunidades de pesquisa, instituição financeira, governo e facilitadores do cluster.

Por fim, há a dinâmica do cluster, que pode ser encarada como os elementos que permitem avaliar a sua "saúde", como a localização, proximidade, concentração e ligações entre os atores; capital social; economias de aglomeração; massa crítica e inovação. Quanto a isso, uma questão 
chave é se o cluster atingiu a massa crítica necessária para sustentar o seu crescimento e desenvolvimento. Também é importante determinar se forças observadas não são meramente os efeitos do desenvolvimento regional natural. A inovação é outro fator-chave: atividades inovadoras são reforçadas dentro do cluster, como resultado da dinâmica.

Paralelamente, Eisingerich et al. (2010), baseados na teoria da rede social, identificaram que o desempenho de um cluster depende da (a) força da rede e da (b) abertura da rede. Força da rede envolve a frequência, a intensidade, a estabilidade de interações e os níveis de confiança entre os membros do cluster. A abertura de rede como uma função da diversidade dos seus membros, vontade de aceitar novos membros e a existência de laços com organizações fora do cluster. Como indicam Aziz e Norhashim (2008), os clusters são não apenas onde as organizações produzem bens, mas também podem ser vistos como locais propícios para o surgimento de novas empresas.

Os efeitos da força e da abertura da rede no desempenho são moderados pela incerteza ambiental. Especificamente, os efeitos positivos da abertura da rede sobre o desempenho do cluster tendem a aumentar à medida que aumenta a incerteza do ambiente, enquanto que os efeitos positivos da força de rede sobre o desempenho do aglomerado tendem a diminuir à medida que aumenta a incerteza ambiental.

Conforme Eisingerich et al. (2010), a velocidade e facilidade com que as inovações podem ser detectadas dentro de clusters provavalmente dependam da diversidade de atores, a abertura a novos membros e a extensão de ligações com organizações que operam fora do cluster. Por outro lado, o grau em que as redes são de confiança e coesas permitirão que as organizações alavanquem tais inovações. Consequentemente, redes fortes em ambientes incertos irão fornecer um papel complementar e de apoio. Em mercados estáveis, no entanto, em que laços fortes de rede tendem a ser mais valiosos; redes fortes fornecem o contexto ideal para explorar inovações existentes e buscar eficiências econômicas.Ou seja, tanto a força de rede quanto seu nível de abertura podem ser benéficas aos clusters, mas sob diferentes condições ambientais: a capacidade das empresas para gerir o equilíbrio entre a força e abertura é uma chave para o cluster sustentável.

Tendo em vista tais processos relacionados ao desempenho de um cluster, a pesquisa de Belso-Martínez, Molina-Morales e Mas-Verdu (2011) mostra que as empresas com melhores recursos internos exploram melhor os recursos externos. No entanto, reforça que a relevância dos recursos internos e capacidades não deve subestimar os recursos externos, aqueles que vêm de relações externas ou de rede. Assim, as novas empresas em um cluster não devem exclusivamente desenvolver seus recursos internos e capacidades, mas devem, consequentemente aproveitá-los para se beneficiar das externalidades derivadas do cluster. 
Fabiano Larentis, Rafael Giovanella \& Tatiane Pellin Cislaghi

Por fim, considerando clusters bem sucedidos, diversos são os estudos que indicam seus fatores de sucesso. Desta forma, com base nos autores Rosenfeld (1997), Porter (1998), assim como Giuliani e Bell (2005) e Bell (2005), são apontadas algumas características que podem explicar seus resultados (Quadro 1), divididas em aspectos estruturais; estratégicos e de mercado, de inovação e tecnológicos; e sociais e relacionais:

\begin{tabular}{|c|c|}
\hline ASPECTOS & CARACTERÍSTICAS \\
\hline $\begin{array}{l}\text { Estruturais } \\
\text { do cluster }\end{array}$ & $\begin{array}{l}\text { - Elementos de suporte desenvolvidos (assistência técnica e gerencial, P\&D, fontes de } \\
\text { subcontratação, logística, telecomunicações, educação, agências regulatórias, sistemas de } \\
\text { financiamento, centros de pesquisa e universidade, entrepostos de comercialização); } \\
\text { - Estrutura que adota todos os clusters em uma nação ou estado; } \\
\text { - Fronteiras apropriadas (que podem ir além das fronteiras políticas). }\end{array}$ \\
\hline $\begin{array}{c}\text { Estratégicos } \\
\text { e de } \\
\text { mercado }\end{array}$ & $\begin{array}{l}\text { - Compreensão compartilhada de competitividade na construção de vantagem competitiva; } \\
\text { - Energia empreendedora (atração de novas empresas, necessárias para a continuação do cluster), } \\
\text { Foco em remover obstáculos para a atualização dos clusters; } \\
\text { - Forte foco no mercado e nos consumidores; } \\
\text { - Papel ativo não somente do setor privado, mas também do governo; } \\
\text { - Visões compartilhadas e institucionalização (esforço de longo prazo, visão de futuro). }\end{array}$ \\
\hline $\begin{array}{l}\text { Ligados à } \\
\text { inovação }\end{array}$ & $\begin{array}{l}\text { - Capacidade absortiva (de absorver, difundir e explorar conhecimento extra-cluster); } \\
\text { - Força de trabalho com conhecimentos tácitos e explícitos de uma indústria. }\end{array}$ \\
\hline $\begin{array}{l}\text { Sociais e } \\
\text { relacionais }\end{array}$ & $\begin{array}{l}\text { - Alto nível de capital social que liga os membros do cluster e fornece oportunidades para interação } \\
\text { informal e aprendizado; } \\
\text { - Amplo envolvimento dos participantes dos clusters e instituições associadas; } \\
\text { - Atenção a relacionamentos pessoais; } \\
\text { - Cooperação para manter o cluster e desenvolver a região; } \\
\text { - Empresas conscientes dos benefícios de suas interdependências; } \\
\text { - Interfaceadores (boundary spanners) atuantes entre as fronteiras das empresas e na fronteira do } \\
\text { cluster; } \\
\text { - Intensidade de networking (nível de cooperação - taxa de networking geralmente reflete os níveis } \\
\text { de capital social e confiança que existe. }\end{array}$ \\
\hline
\end{tabular}

Quadro 2 - Características de clusters bem sucedidos

Verifica-se que esses fatores de sucesso estão relacionados a elementos da existência e da efetividade de instituições associadas ao cluster, de foco em resultado e no longo prazo e forte interação e relacionamento dos integrantes do cluster. Isso indica que o sucesso de clusters depende, além de elementos econômicos, de dimensões sociais e de relacionamento, como confiança, cooperação, aprendizagem e valores compartilhados. Isso tanto dentro do cluster quanto entre o 
cluster e as comunidades em que ele está localizado, assim como na relação com outros stakeholders. Identificam-se, portanto, elementos de rede e de sustentabilidade presentes em clusters de sucesso.

\section{CLUSTER E SUA SUSTENTABILIDADE}

Clusters, como concentração de empresas que, em função dela, podem adquirir vantagens competitivas, estão inseridos em regiões específicas, ou seja, afetam os habitantes e os recursos inseridos nelas. Repercute, portanto, não somente em questões econômicas e competitivas, mas também em questões sociais e em questões ambientais. Gera influências, portanto, no desenvolvimento sustentável dessa região. Em relação a isso, Siqueira, Serrano, Rimonato e Tartareli (2011) reforçam que a identificação da capacidade dos clusters para estimular o desenvolvimento da economia de uma região ou país tem levado políticos, agências de desenvolvimento e governos a se interessar por eles, reconhecendo-os como uma opção para estimular e ampliar a competitividade de indústrias, de reduzir desigualdades entre regiões ou de criar polos de desenvolvimento.

De acordo com Porter (1998), na economia, leva-se em consideração o cluster, a natureza da competição e o papel da localização na construção de vantagem competitiva. A saúde do cluster, portanto, para ele, é importante para a saúde da empresa. Aqui fica evidente a perspectiva econômica da sustentabilidade do cluster para o desenvolvimento sustentável das empresas que o compõem.

Por outro lado, conforme Rosenfeld (1997), deve-se tratar economias locais como sistemas de produção e sistemas sociais. Cada negócio depende, assim, de suas ligações com outros negócios, com agências não-governamentais sem fins lucrativos e governamentais para vendas e compras, recursos, informação e ideias. O cluster, nesse contexto, é representado por um sistema de produção local que está incorporado em um sistema social local. Ou seja, pode-se vislumbrar aqui a necessidade de se avaliar o desenvolvimento sustentável econômico e social dentro de clusters, e do elemento social acima do elemento econômico. Mesmo não evidente a perspectiva ambiental, afirma-se que esta também repercutirá no futuro da região e de seus habitantes, uma vez que usufrui de seus recursos naturais.

Paralelamente, no estudo de Zettinig e Vineze (2012), três processos críticos impulsionam o desenvolvimento sustentável de um cluster: a utilização (exploitation) das oportunidades atuais, a 
Fabiano Larentis, Rafael Giovanella \& Tatiane Pellin Cislaghi

exploração (exploration) de oportunidades futuras e os processos que facilitam o equilíbrio entre esses dois elementos. Tais processos dependem da definição de estratégias, da integração de estruturas, atividades e relações internas e da adaptação do cluster a pressões, eventos e tendências externas, que por sua vez afetarão a configuração de integração entre seus membros.

Os autores ainda afirmam a importância, para a gestão e financiamento do cluster, da existência de mecanismos de equilíbrio que ajudam a assegurar o desenvolvimento sustentável. Ou seja, mecanismos de coordenação, ou de governança, com foco na viabilidade sustentável, que requerem a gestão crítica do balanço entre os processos de utilização e de exploração. Tais mecanismos podem assumir diversas formas, como por exemplo através de uma entidade legal de propriedade conjunta dos membros, e ser iniciada por vários atores, como por exemplo a partir de uma política pública.

Além disso, Breznitz (2013) afirma que a inovação pode ser a base de um setor, mas pode não ser suficiente para a sua sustentabilidade. Nesse caso, uma das razões para a falta de desenvolvimento do cluster é a falta de base de conhecimento e de experiência em todas as fases de produção. Especificamente em relação a clusters ligados à pesquisa, inovação e desenvolvimento, o autor identificou a importância da existência de empresas maduras que fornecem conhecimento e experiência, de funcionários experientes e da possibilidade de adquirir fases posteriores da produção em uma pequena base tecnológica.

Paralelamente, o modelo de Cunha e Cunha (2005), baseado na teoria de clusters e com o propósito de avaliar o impacto da atividade de turismo no desenvolvimento de uma região, integra a competitividade sistêmica e conceitos de sustentabilidade nas dimensões econômicas, sociais, culturais, ambientais e institucionais/políticas. A competitividade sistêmica compreende quatro níveis de relações (meta, macro, meso e micro), que interferirão no desenvolvimento sustentável de um cluster. A meta competitividade engloba fatores sócio-culturais que definem a capacidade de articulação dos atores sociais para formulação de estratégias e políticas de interesses da sociedade local. A macro competitividade deriva de estratégias macroeconômicas e de estabilidade definidas pelas políticas fiscal, monetária, de câmbio, comercial e da concorrência. A meso competitividade envolve estruturas de suporte que facilitam a interação e cooperação entre empresas (fornecedores, clientes e concorrentes), instituições de pesquisa e inovação, de suporte financeiro, instituições de apoio e de comercialização, de formação e treinamento e infra-estrutura (Transportes, comunicações e energia). A micro competitividade, por sua vez, é a capacidade de uma empresa ou rede da empresas ser competitiva, oferecendo bens e serviços que otimizem a relação custoeficiência, qualidade e variedade, podendo responder por novas oportunidades de mercado e mudanças. 
O desafio, entretanto, como já colocado quando se comentou sobre estratégias sustentáveis, é considerar essas dimensões do desenvolvimento sustentável em conjunto, avaliando seus efeitos a longo prazo, incluindo as gerações futuras. Neste caso, considerando clusters, a complexidade aumenta, uma vez que não há menção apenas a uma empresa, mas a diversas empresas, concentradas e influenciando, dentro de uma região, as vidas de diversas pessoas, assim como as políticas públicas e o meio ambiente.

A título de exemplificação, Larreina e Aguado (2008) identificaram a importância do cluster vinícola para a economia da região de Rioja, na Espanha. O aumento das vendas de vinho na região é simultânea à expansão do bem-estar entre os habitantes da região. A explicação para a relação entre bem-estar regional e desempenho positivo do setor do vinho de Rioja está no grande número de famílias que dependem dele.

Uma sugestão para isso é enxergar o cluster de uma perspectiva de rede (mesmo que conceitualmente pode não ser considerado uma rede de natureza cooperativa), e prover meios para que elementos dessa perspectiva sejam desenvolvidos. Como uma perspectiva de rede considera interação a longo prazo e confiança, esses elementos, de caráter social, os quais podem promover maior respeito entre os envolvidos e maior consideração dos efeitos das suas ações no futuro dos seus relacionamentos, também podem gerar respeito e maior consideração do clusters quanto a seus stakeholders.

Além disso, percebe-se que para compreender, avaliar e influenciar o desenvolvimento e desempenho de um cluster, dependente tanto das empresas que o compõem, assim como de seus stakeholders, como governos e comunidades, e de seus mecanismos de governança, deve-se levar em conta a lógica do desenvolvimento sustentável e das estratégias sustentáveis, dentro de um olhar complexo. Por outro lado, as três dimensões que compõem a sustentabilidade, acrescentando a quarta dimensão (de segunda ordem), proposta por Steurer, et al. (2005) e as dimensões culturais e institucionais/políticas, indicadas por Cunha e Cunha (2005), podem ser identificadas, mesmo que de forma isolada, em clusters bem sucedidos. O modelo conceitual, apresentado a seguir, espera contribuir com tais reflexões e desafios.

\subsection{MODELO CONCEITUAL}

Tendo em vista o exposto anteriormente, no que foi abordado nas seções sobre redes, sobre cluster e sobre a sua relação com sustentabilidade, segue modelo conceitual (Figura 1). Apresentado em formato de espiral pela natureza processual, complexa e em rede das dimensões consideradas, $o$ modelo, que tem por intuito compreender como se desenvolve a sustentabilidade de um cluster, é 
Fabiano Larentis, Rafael Giovanella \& Tatiane Pellin Cislaghi

dividido em três partes: (a) fatores iniciais, de natureza predominantemente de base ou estrutural; (b) fatores intermediários, de natureza predominantemente ligada à ação; e (c) fatores resultantes, considerando o desempenho e a sustentabilidade do cluster. Além dessas partes, há o contexto, ou seja, elementos externos ao cluster e associados à sua trajetória, que interferem em seu desenvolvimento. Importante ressaltar que, por haver uma espiral, os fatores iniciais interferem tanto nos intermediários quanto nos resultantes. Ademais, tais fatores apresentam interação com o contexto.

Os fatores iniciais são compostos por: (1) atores do cluster, mecanismos de governança, recursos e localização e (2) estratégias do cluster. Tais elementos possuem natureza predominantemente de base ou estruturais, sendo considerados antecedentes.

Os fatores intermediários são compostos por: (3) força e abertura do cluster; (4) processos de integração e de adaptação do cluster; (5) criação/estoque de conhecimento e movimentação do conhecimento; e (6) ações de utilização (exploitation) e exploração (exploration). Esses elementos são de natureza predominantemente ligada a ações e processos, podendo ser considerados como mediadores entre os antecedentes e resultantes.

Os fatores resultantes englobam: (7) desempenho do cluster; e (8) sustentabilidade do cluster. O contexto, por sua vez, envolve os elementos associados à trajetória do cluster (estágio do ciclo de vida e níveis de competitividade) e os elementos de natureza ambiental (nível de incerteza ambiental, demanda dos mercados e mudança tecnológica). Pressupõe-se que as interações de cada fator com o contexto (influências mútuas) gerarão efeitos nos outros fatores. Por exemplo, a interação dos fatores iniciais (de base) com o contexto gerará efeitos também nos fatores intermediários e resultantes.

É a partir dessa base (fator número 1) que o cluster desenvolve e define suas estratégias (fator número 2 - Zettinig \& Vineze, 2012), as quais darão direcionamento às ações do cluster. Como observações ao primeiro nível de fatores, apesar da estratégia e dos mecanismos de governança apresentar também caráter processual e de ação, foram classificados nos fatores iniciais por sua importância aos fundamentos, diretrizes e coordenação de um cluster. Em relação à localização, apesar de poder ser classificada dentro de recursos, optou-se por apresentá-la como um fator isolado, por sua importância dentro da abordagem de clusters. 
Figura 1 - Modelo conceitual proposto

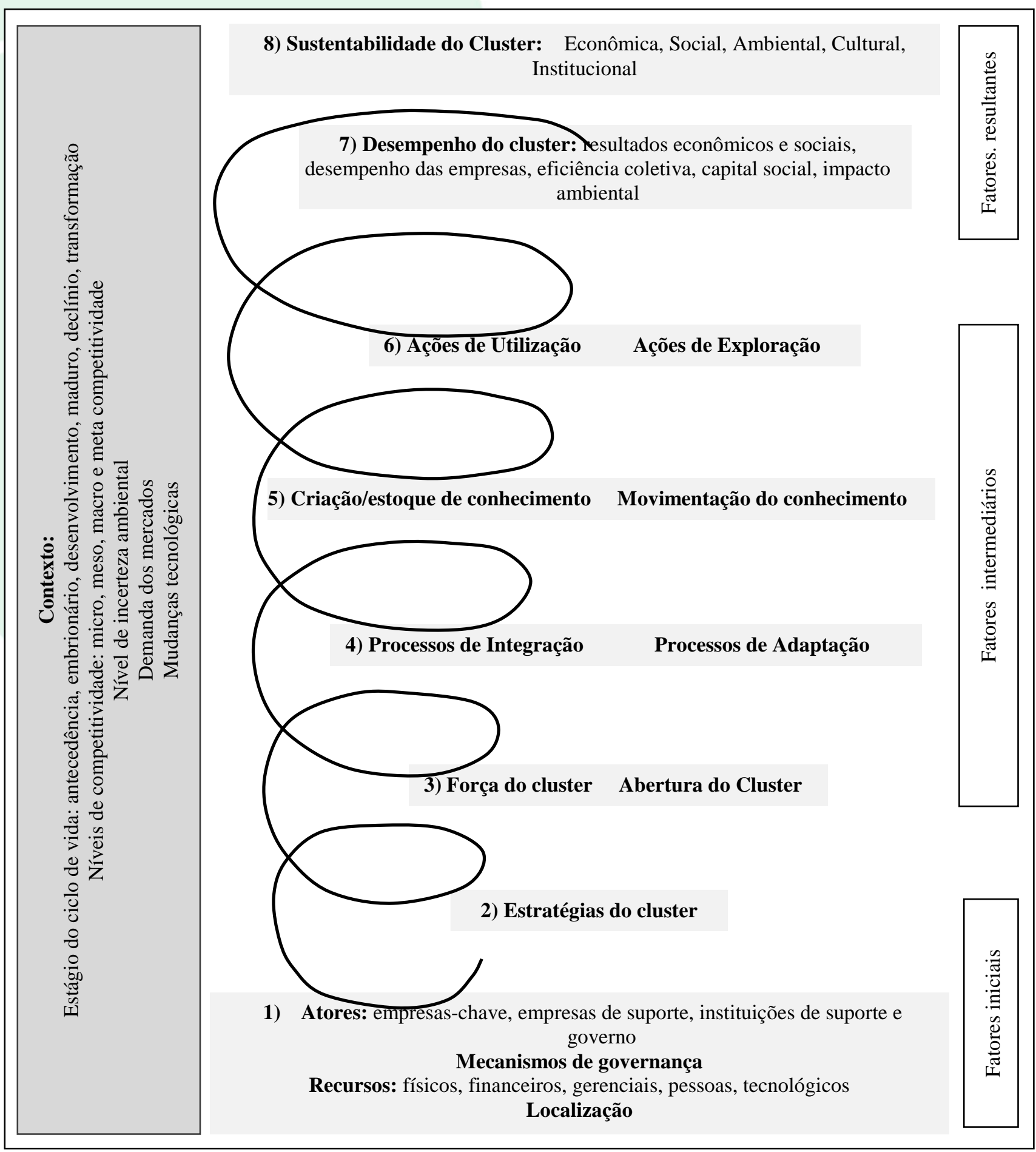

O primeiro conjunto de fatores do nível intermediário é composto pela força e pela abertura do cluster (número 3), apresentado por Eisingerich et al., (2010). Força envolve a frequência, intensidade, estabilidade de interações e níveis de confiança entre os membros do cluster. Abertura envolve a diversidade dos seus membros, vontade de aceitar novos membros e a existência de laços 
Fabiano Larentis, Rafael Giovanella \& Tatiane Pellin Cislaghi

com organizações fora do cluster. Pressupõe-se que são dependentes e resultantes dos fatores de base do cluster.

O segundo conjunto de fatores do nível intermediário (número 4) envolve processos de integração e adaptação (Zettinig \& Vineze, 2012). A integração é de estruturas, atividades e relações internas do cluster e a sua adaptação a pressões, eventos e tendências externas a ele. Entende-se que ambos fatores sejam influenciados pela força e pela abertura do cluster, por estes envolverem graus de interações e cooperação, assim como nível de diversidade e entrada de novos membros (aspectos internos e externos). Na integração e de adaptação colocam-se em ação os elementos constituintes da força e abertura do cluster, assim como os direcionamentos da estratégia e os atores, governança, recursos e localização.

O terceiro conjunto envolve a criação e estoque de conhecimento e a movimentação de conhecimento (número 5), apresentados por Aziz e Norhashim (2008). A criação e estoque de conhecimento é um dos aspectos que determina o nível de inovação, avaliado a partir de atividades e investimentos em pesquisa e desenvolvimento. A movimentação é composta pela interação e cooperação dos atores dentro do cluster. Entende-se que ambos fatores sejam influenciados pela força e pela abertura do cluster, assim como pelos processos de integração e adaptação, por estes envolverem relações internas e adaptações a tendências e pressões externas, os quais geram necessidade de informações e de conhecimento.

O quarto conjunto (número 6) envolve ações de utilização e exploração (Zettinig \& Vineze, 2012). A utilização é das oportunidades atuais e a exploração das oportunidades futuras, que dependem, conforme os autores, da integração e da adaptação do cluster. Pode-se afirmar também que tais ações ocorrerão tendo por fundamento os resultados da criação, do estoque e da movimentação de conhecimento.

Após os fatores intermediários seguem os fatores resultantes: desempenho do cluster e sustentabilidade do cluster. O desempenho é baseado no estudo de Carpinetti, Galdámez e Gerolamo (2008) e as dimensões de sustentabilidade no trabalho de Cunha e Cunha (2005). Depreende-se, com base em Eisingerich et al. (2010), que o desempenho do cluster definirá o nível e qualidade de sua sustentabilidade. Convém lembrar, neste caso, que o desempenho do cluster foi considerado como variável dependente no modelo de Aziz e Norhashim (2008), recebendo influência de sua força e abertura (Eisingerich et al., 2010) e da criação/estoque e movimentação do conhecimento (Aziz \& Norhashim, 2008).

No que tange ao contexto, foram considerados os estágios do ciclo de vida de Aziz e Norhashim (2008), os níveis de competitividade (micro, meso, macro e meta - Cunha \& Cunha, 2005), o nível de incerteza, moderando a relação de força e abertura com desempenho do cluster 
(Eisingerich et al., 2010), a demanda (Huggins, 2008; Porter, 1998; Tödtling, 1994) e as mudanças tecnológicas, visto a sua importância no desenvolvimento e sustentabilidade do cluster (Aziz \& Norhashim, 2008; Calamel et al., 2012; Porter, 1998). Entende-se que a configuração da espiral dependerá da situação e momento em que o cluster se encontra enquanto ciclo de vida e competitividade, da incerteza ambiental, indicando maior ou menor nível de turbulência, da demanda e necessidades dos clientes e consumidores, assim como da forma como as tecnologias associadas a ele vêm evoluindo. Por outro, alerta-se que a espiral não apresenta papel passivo em suas relações com o contexto. Em relação a isso, ressalta-se, em relação à sustentabilidade, que mesmo aparecendo como o oitavo fator do modelo, depende dos anteriores e do contexto para se desenvolver.

Desta forma, tendo em vista que o pensamento complexo, contrário à inteligência parcelada e reducionista, pode esclarecer as estratégias do mundo incerto (Morin \& Moigne, 2000), e que "lentes" tradicionais, baseadas na lógica econômico-financeira, não dão conta suficientemente da compreensão e da prática do desenvolvimento sustentável, uma vez que o paradigma da complexidade procura introduzir uma análise interdisciplinar do fenômeno (Cruz et al., 2006), pode-se afirmar que o fenômeno dos clusters, ainda mais quando associado às abordagens da sustentabilidade, é multifacetado. Necessita, em função disso, que uma análise que se origine do pensamento complexo. O modelo proposto, baseado na relação entre clusters, redes e sustentabilidade, e evidenciado nas interações entre a espiral e o contexto, parte desse pressuposto.

\section{CONSIDERAÇÕES FINAIS}

O cluster é um fenômeno interorganizacional complexo e multifacetado, influenciado e com influência sobre seus stakeholders. Tem responsabilidade não somente no aspecto econômico, tanto das empresas que o compõem quanto da região em que está inserido, mas também em outros resultados. Gera empregos, permite renda e um nível de qualidade de vida às pessoas de determinada região, mas utiliza os seus recursos naturais.

O conceito de cluster, de uma maneira abrangente, pode ser definido como concentração setorial e geográfica de empresas. Dentre as características mais importantes tem-se o ganho de eficiência coletiva, sendo importante ressaltar que os clusters são formados apenas quando os aspectos setorial e geográfico estão concentrados. Em um cluster encontra-se um amplo escopo para a divisão de tarefas entre empresas, bem como para a especialização e para a inovação, elementos essenciais para a competição além de mercados locais (Olave \& Neto, 2001). No entanto, é 
Fabiano Larentis, Rafael Giovanella \& Tatiane Pellin Cislaghi

importante avaliar se o cluster atingiu a massa crítica necessária para sustentar o seu crescimento e desenvolvimento (Aziz \& Norhashim, 2008).

A sustentabilidade, por considerar um foco a longo prazo, uma visão de futuro não é apenas econômica, mas também social, ambiental, cultural e política. Em função disso, deve-se considerar, cada vez mais, estratégias de caráter sustentável, não somente de uma ótica econômica. Pode-se fazer menção a vantagens competitivas sustentáveis, não somente levando em consideração a diferenciação e a dificuldade dos concorrentes em copiar ou em oferecer algo equivalente, mas de levar em consideração essa competitividade (lógica econômica) juntamente com questões sociais e ambientais.

Colocar em prática o desenvolvimento sustentável e as estratégias sustentáveis é ainda visto como um desafio para muitas empresas, inclusive àquelas que efetuam ações relacionadas à sustentabilidade, muitas vezes isoladas e descontextualizadas. Na dimensão de um cluster adquire uma maior complexidade, uma vez que aqui se considera fundamentalmente uma região, uma concentração de empresas em interação e, em decorrência, as vidas de diversas pessoas, as ações dos governos e de outras instituições envolvidas. Afinal, baseado em Giuliani e Bell (2005), o cluster não dever ser somente localizado e concentrado (elemento geográfico e setorial), mas também ser sentido como tal.

Este ensaio teórico teve por objetivo propor um modelo conceitual, derivado da discussão acerca da relação entre cluster e sustentabilidade. Tal modelo, sustentado por três níveis arranjados em espiral e por aspectos contextuais, pode servir de subsídio para compreender melhor as possíveis relações entre a constituição e desenvolvimento de um cluster e sua sustentabilidade, que vai além de aspectos econômicos.

A fim de alcançar um desenvolvimento sustentável conjunto, os gestores e formuladores de políticas de cluster precisam entender também o desempenho do mesmo. Um cluster envolve diversas empresas de diferentes níveis econômicos, concentradas em um região. Ainda, é dependente dessa região para poder se perpetuar, poder gerar vantagens competitivas. Por outro lado, o desenvolvimento regional é influenciado pelo desenvolvimento de clusters. Acredita-se que o modelo conceitual em questão contribua para o entendimento de tais desafios.

Nesse sentido, como já colocado, abordar o desenvolvimento de um cluster, sob o prisma das abordagens de redes e da sustentabilidade, pode ser uma forma de encarar esse desafio: interações sociais e bons resultados econômicos com respeito ao outro, ao ambiente e às gerações futuras. Tal com indicam Reid, Smith e Carroll (2008), como a abordagem de rede leva em conta como base as relações sociais ou interpessoais, ela fornece uma dimensão que as técnicas com foco em relações econômicas não captam. 
Como sugestão para futuros estudos, propõe que o modelo conceitual seja avaliado e validado empiricamente, a partir de abordagens metodológicas múltiplas (qualitativa e quantitativa). Recomenda-se primeiramente uma pesquisa qualitativa, tendo por intuito a análise das relações entre os fatores e destes com o contexto, incluindo a avaliação da sequência proposta de oito elementos. Posteriormente, uma pesquisa quantitativa, com o desenvolvimento de escalas de mensuração a partir da literatura e dos achados da pesquisa qualitativa, com o intuito de validar e confirmar os fatores propostos e suas relações de interdependência, recomendando-se o uso da Modelagem de Equações Estruturais. Sugere-se, em relação a essas questões de ordem metodológica, a escolha de clusters em diferentes contextos, envolvendo a tecnologia dominante e suas tendências, a incerteza ambiental, as características da competitividade e da demanda, assim como os seus ciclos de vida.

\section{REFERENCIAS}

Aquino, A. L., Bresciani, L. P. (2005). Arranjos produtivos locais: uma abordagem conceitual. Organizações em Contexto, 1 (2), 153-167.

Aziz, K. A., Norhashim, M. (2008) Cluster-based policy making: assessing performance and sustaining competitiveness. Review of Policy Research, 25(4), 349-375

Bansal, P., Roth, K. (2000) Why companies go green: a model of ecological responsiveness. Academy of Management Journal, 43(4), 717-736.

Baptista, R., Swann, P. (1998) Do firms in clusters innovate more? Research Policy, 27(5), 525540.

Bell, G. G. (2005) Research notes and commentaries: clusters, networks and firm innovativeness. Strategic Management Journal, 26(3), 287-295.

Belso-Martínez, J. A., Molina-Morales, F. X., Mas-Verdu, F. (2011) Clustering and internal resources: moderation and mediation effects, Journal of Knowledge Management, 15 (5), 738 758 
Fabiano Larentis, Rafael Giovanella \& Tatiane Pellin Cislaghi

Benites, L. L. L., Pólo, E. F. (2013) A sustentabilidade como ferramenta estratégica empresarial: governança corporativa e aplicação do Triple Bottom Line na Masisa. Revista de Administração da UFSM, 6 (Edição Especial), 827-841.

Breznitz, S. M. (2013) Cluster Sustainability: The Israeli Life Sciences Industry. Economic Development Quarterly 2013 27(1), 29-39

Britto, J., Stallivieri, F. (2010) Inovação, cooperação e aprendizado no setor de software no Brasil: análise exploratória baseada no conceito de Arranjos Produtivos Locais (APLs). Economia e Sociedade, 19 (2), 315 - 358.

Calamel, L., Defélix, C., Picq, T., Retour, D. (2012) Inter-organisational projects in French innovation clusters: the construction of collaboration. International Journal of Project Management, 30 (1), 48-59.

Carpinetti, L. C. R., Galdámez, E. V. C., Gerolamo, M. C. (2008), A measurement system for managing performance of industrial clusters: a conceptual model and research cases. International Journal of Productivity and Performance Management, 57(5), 405 - 419.

Chung, W. W. C., Yam, A. Y. K., Chan, M. F. S. (2004) Networked enterprise: a new business model for global sourcing. International Journal of Production Economics, 87(3), 267-280.

Cruz, L. B., Pedrozo, E. Á., Estivalete, V. F. B. (2006) Towards sustainable development strategies: a complex view following the contribution of Edgar Morin. Management Decision, 44(7), 871891.

Cunha, S. K., Cunha, J. C. (2005) Tourism cluster competitiveness and sustainability: proposal for a systemic model to measure the impact of tourism on local development. $B A R, 2(2), 47-62$.

Eisingerich, A. B., Bell S. J, Tracey, P. (2010) How can clusters sustain performance? The role of network strength, network openness, and environmental uncertainty. Research Policy, 39(2), 239-253

Felzensztein, C., Gimmon, E., Aqueveque, C. (2012) Clusters or un-clustered industries? Where inter-firm marketing cooperation matters". Journal of Business \& Industrial Marketing, 27(5), $392-402$

Feser, E. J. (1998). Old and new theories of industry clusters. In M. Steiner (Ed.), Clusters and regional specialisation (pp. 18-40). London: Pion.

Figueiredo, J. C; Di Serio, L. C. (2007) Estratégia em clusters empresariais: conceitos e impacto na competitividade. In: Di Serio, L. C. (Org). Clusters empresariais no Brasil: casos selecionados. São Paulo: Saraiva. 
Galdámez, E. V. C., Carpinetti, L. C. R., Gerolamo, M. C. (jan/mar. 2009) Proposta de um sistema de avaliação do desempenho para arranjos produtivos locais. Gestão e Produção, 16 (1), 133151.

Giuliani, E., Bell, M. (2005) The micro-determinants of meso-level learning and innovation: evidence from a Chilean wine cluster. Research Policy, 34(1), 47-68.

Gladwin, T. N., Kennelly, J. J., Krause, T. (1995) Shifting paradigms for sustainable development: implications for management theory and research. Academy of Management Review, 20(4), 874907.

Huggins, R. (2008) The evolution of knowledge clusters: progress and policy. Economic Development Quarterly, 22(4), 277-289.

Hart, S. L. (1995) A natural-resource-based-view of the firm. Academy of Management Review, 20 (4), 986-1014.

Larreina, M., Aguado, R. (2008) Beyond the cluster: how wine drives regional economy to success: "Oenopolis", the case of Rioja". International Journal of Wine Business Research, 20(2), 153 170

Mackey, A.; Mackey, T.; Barney, J. B. (2007) Corporate social responsibility and firm performance: investor preferences and corporate strategies. Academy of Management Review, 32 (3), 817-835.

Marconatto, D. A. B., Trevisan, M., Pedrozo, E. A., Saggin, K. D.;, Zonin, V. J. (2013) Saindo da trincheira do desenvolvimento sustentável: uma nova perspectiva para a análise e a decisão em sustentabilidade. Revista de Administração Mackenzie, 14 (1), 15-43.

Mascena, K. M. C., Figueiredo, F. C., Boaventura, J. M. G. (set/out 2013) Clusters e APLs: Análise Bibliométrica das Publicações Nacionais no Período de 2000 a 2011. Revista de Administração de Empresas, 53(5), 454-468.

Masquietto, C. D., Neto, M. S., Giuliani, A. C. (mai/ago 2010). Identificação de Arranjos Produtivos Locais: o caso do arranjo produtivo local do álcool de Piracicaba. Gestão e Regionalidade, 26 (77), 75-87.

Mauerhofer, V. (2008) 3-D sustainability: an approach for priority setting in situation of conflicting interests towards a sustainable development. Ecological Economics, 64 (3), 496-506.

Mebratu, D. (1998) Sustainability and sustainable development: historical and conceptual review. Environmental Impact Assess Review, 18, 493-520.

Meyer-Stamer, J. (2001) Estratégias de desenvolvimento local e regional: clusters, política de localização e competitividade sistêmica. Policy Paper Friedrich Ebert Stiftung, 28, 6-26 
Fabiano Larentis, Rafael Giovanella \& Tatiane Pellin Cislaghi

Morin, E. (1987) O Método I: A natureza da natureza. Lisboa: Europa-América.

Morin, E., Moigne, J. (2000) A Inteligência da Complexidade. São Paulo: Petrópolis.

Olave, M. E. L. Neto, J. A. (2001) Redes de cooperação produtiva: uma estratégia de competitividade e sobrevivência para pequenas e médias empresas. Gestão \& Produção, 8(3), 289-303.

Omta, S. W. F, Trienekens, J. H., Beers, G. (2001) Chain and network science: a research framework. Journal of Chain and Network Science, 1(1), 1-6.

Pedrozo, E. Á., Hansen, P. B. (2001) Clusters, filière, supply chain, redes flexíveis: uma análise comparativa. Análise, 12(2), 7-19.

Perry, M. (2005) Business clusters: an international perspective. New York: Routledge.

Polodny, J. M.; Page, K. L. (1998) Network forms of organization. Annual Review of Sociology, 24, 57-76.

Porter, M. (1998) On competition. Boston: Harvard Business Review Book.

Quandt, C. O. (2012) Redes de cooperação e inovação localizada: estudo de caso de um arranjo produtivo local. Revista de Administração e Inovação, 9 (1), 141-166.

Reid, N., Smith, B. W., Carroll, M. C. (2008) Cluster regions: A social network perspective. Economic Development Quarterly, 22(4), 345-352.

Roelandt, T. J. A., Hertog, P. D. (1999) Cluster analysis and cluster-based policy making: The state of the art. In OECD (Ed.). Boosting innovation: The cluster approach (pp. 413-427). Paris: OECD.

Rosenfeld, S. A. (1997) Bringing business clusters into the mainstream of economic development. European Planning Studies, 5(1), 3-23.

Schaltegger, S., Wagner, M. (2011) Sustainable entrepreneurship and sustainability innovation: categories and interactions. Business Strategy and the Environment, 20(4), 222-237.

Siqueira, J. P. L. , Serrano, D. P. , Rimonato, I. P. O. S., Tartareli, R. (2011) Uma avaliação da produção acadêmica brasileira recente sobre clusters de negócios Revista Ibero-Americana de Estratégia - RIAE, 10 (1), 55-76.

Steurer, R., Langer, M. E., Konrad, A., Martinuzzi, A. (2005) Corporations, stakeholders and sustainable development I: a theoretical exploration of business-society relations. Journal of Business Ethics, 61 (3). 263-281. 
Tödtling, F. (1994) Regional networks of high-technology firms: The case of the greater Boston region. Technovation, 14(5), 323-343.

Verschoore, J. R. Balestrin, A. (2008) Ganhos competitivos das empresas em redes de cooperação. Revista de Administração USP - Eletrônica, 1(1), 1-21.

Zaccarelli, S. B.. Telles, R.. Siqueira, J.P.L.. Boaventura, J.M.G.. Donaire, D. (2008) Clusters $e$ redes de negócios: uma nova visão para a gestão dos negócios. São Paulo: Atlas.

Zettinig P., Vincze, Z. (2012) How clusters evolve. Competitiveness Review: An International Business Journal incorporating Journal of Global Competitiveness, 22(2). 110 - 132.

Recebido: 30/03/2013

Aprovado: 27/05/2013 